\title{
Complex Logarithmic Number System Arithmetic Using High-Radix Redundant CORDIC Algorithms
}

\author{
David Lewis \\ Department of Electrical and Computer Engineering, University of Toronto \\ Toronto, Ontario, Canada M5S 3G4 \\ lewis@eecg.toronto.edu
}

\begin{abstract}
This paper describes the application of high radix redundant CORDIC algorithms to complex logarithmic number system arithmetic. It shows that a CLNS addition can be performed with approximately the same hardware as a high-radix CORDIC operation. A design example comparable to single precision floating point has been designed and simulated.
\end{abstract}

\section{Introduction}

Logarithmic number system (LNS) representation has been the subject of considerable theoretical interest since its introduction [10], and a number of implementations described, e.g. [7]. Arnold recently described arithmetic transformations for efficient software implementations, as well as pointing out the advantages of complex valued LNS (CLNS) [11]. CLNS is potentially attractive in areas such as FFTs, where the powers of unity have exact representation, and complex multiplications can be easily performed using fixed point additions. However, previous VLSI implementations of LNS rely on interpolation of a function of a single variable and do not extend to CLNS. Compared to floating point representation, where a complex multiply requires 4 FP multiplies and 2 FP adds, a CLNS multiply requires only 2 fixed point adds. Consequently, if the cost of a CLNS add can be reduced below 4 FP multiplies and 4 FP adds, the total cost of a CLNS multiply-add will be less than FP.

CORDIC algorithms have long been advocated for trigonometric functions as well as complex valued exponentials and logs [1] [2] [3]. Most efforts in CORDIC have focused on real numbers, and used low radix-2 or radix-4 algorithms. Recently, BKM, a low-radix redundant CORDIC algorithm was described and used for trigonometric functions and complex arithmetic using a linear representation [8]. BKM, as most other CORDIC algorithms is a low radix method, and takes many steps to perform an operation. The simplicity of the hardware implementation of CORDIC is attractive, and a number of successful hard- ware implementations of CORDIC have been also been described [4] [5] [6] [9], however, these typically take a large number of stages. A few high radix methods have been described. Baker [12] described high radix CORDIC based algorithms, later extended to carry-save representation by Antelo et al [15]. Ahmed [13] [14] introduced a convergence method that generalized Chen's [3], useful for describing algorithms as transformations on numbers that maintain some invariant. Ahmed described CORDIC algorithms using a single high-radix step to begin, and also using linear interpolation for the latter half of the algorithm.

This paper is most closely related to Antelo's et. al highradix CORDIC algorithm [15]. It applies to CLNS, and modifies this algorithm, as well as introducing some optimizations specific to CLNS that approximately halve the cost of the algorithm. Some specific points of comparison to [15] are: (1) this paper shows how optimizations specific to CLNS can eliminate approximately half the CORDIC stages (2) this paper advocates exact calculation of the minimal usable radix, instead of using a fixed radix (3) this paper extends the high-radix algorithms to include logarithm algorithms, similar to CORDIC vectoring, which requires more complex digit selection and a different sequence of operations.

The remainder of this paper describes the number representation assumed for CLNS, and the transformations that can be performed on these numbers. Hardware structures for high-radix operations are described for complex exponentiation and logarithm, together with bounds on the values at each stage. An example processor has been designed and verified down to the gate level, and its verification is described.

\section{Number Representation}

A complex valued number $X=X_{R}+X_{I} \cdot i$ is represented in CLNS by its logarithm, $x=x_{L}+x_{\theta} \cdot i$, such that $X=b^{x}$, where $b$ is the base of the system. Both $x_{l}$ and $x_{\theta}$ are fixed point numbers and can be represented 
using 2's complement binary numbers.

Given the representations $\left\langle x_{L}, x_{\theta}\right\rangle, x=x_{L}+x_{\theta} \cdot i$, and $\left\langle y_{L}, y_{\theta}\right\rangle$ of two numbers $X$ and $Y$, it is trivial to find the representation $\left\langle z_{L}, z_{\theta}\right\rangle$ of $Z=X \times Y \quad$ as $z_{L}=x_{L}+y_{L}$ and $z_{\theta}=x_{\theta}+y_{\theta}$.

CLNS addition is considerably more difficult. To compute the representation of $Z=X+Y$, it is necessary to compute $z_{l}$ and $z_{\theta}$.

$$
\begin{aligned}
& z_{l}=x_{L}+f_{L}\left(x_{L}-y_{L}, x_{\theta}-y_{\theta}\right) \\
& z_{\theta}=x_{\theta}+f_{\theta}\left(x_{L}-y_{L}, x_{\theta}-y_{\theta}\right)
\end{aligned}
$$

The functions $f_{L}$ and $f_{\theta}$ are implicitly defined in terms of $r$, as

$$
\begin{gathered}
r=x-y=x_{L}-y_{L}+\left(x_{\theta}-y_{\theta}\right) \cdot i \\
f(r)=f_{L}(r)+f_{\theta}(r) \cdot i \\
f(r)=\log _{b}\left(1+b^{r}\right)
\end{gathered}
$$

We will assume that $x_{L} \geq y_{L}$ so that the argument to $f_{L}$ and $f_{\theta}$ lies in the right hand half plane. Subtraction is accomplished by adding $\log _{b}(-1)$ to the appropriate operand.

\subsection{Transformations on Complex Number Repre- sentations}

In order to compute $f(r)$ as defined in (5), CORDICbased algorithms can be applied to the computation of the complex exponential and logarithm functions. As in previous descriptions of convergence methods, we define a function that maintains a constant value through each stage in the transformation. Our 4-tuple contains a Cartesian representation of a point using the pair of real values $u_{j}$ and $v_{j}$, and a polar logarithmic representation using the two real values $r_{j}$ and $a_{j}$. The value represented is $T_{j}=\left(u_{j}+v_{j} \cdot i\right) \times b^{r_{j}+a_{j} \cdot i}$. Two transformations, scaling and rotation, are define such that the value of $T_{j}$ is kept constant.

The scale transformation performs a linear scaling of the Cartesian value by a factor of $\left(1+s_{j}\right)$, and compensating reduction in the logarithmic value of $\log _{b}\left(1+s_{j}\right)$ :

$$
u_{j+1}=u_{j} \times\left(1+s_{j}\right)
$$

$$
\begin{gathered}
v_{j+1}=v_{j} \times\left(1+s_{j}\right) \\
r_{j+1}=r_{j}+l_{j} \\
a_{j+1}=a_{j} \\
l_{j}=-\log _{b}\left(1+s_{j}\right)
\end{gathered}
$$

The rotation transformation performs a rotation of the Cartesian values based on some value $q_{j}$ and compensating change in the angle of the polar logarithmic representation:

$$
\begin{gathered}
u_{j+1}=u_{j}+q_{j} \times v_{j} \\
v_{j+1}=v_{j}-q_{j} \times u_{j} \\
r_{j+1}=r_{j}+m_{j} \\
a_{j+1}=a_{j}+c_{j} \\
m_{j}=-\log _{b}\left(\sqrt{1+q_{j}^{2}}\right) \\
c_{j}=\frac{-\operatorname{atan}\left(q_{j}\right)}{\ln (b)}
\end{gathered}
$$

The angle of the rotation is given by $-c_{j}$. The rotation lengthens the vector by a factor of $\sqrt{1+q_{j}^{2}}$, and the corresponding change in the polar logarithm magnitude is given by $m_{j}$. Both the scale transformation and the rotation transformation preserve the invariant $T_{j+1}=T_{j}$.

Complex exponentiation and complex logarithm are implemented using a sequence of rotation and scaling transformations. In each, a series of $N$ stages are cascaded, each of which may be a rotation or scaling transformation according to the design of the algorithm. The inputs are $u_{0}, v_{0}, r_{0}$, and $a_{0}$, and the outputs are $u_{N}, v_{N}, r_{N}$, and $a_{N}$. In each algorithm, some of the inputs and outputs are constrained to be constants. Thus, the difference in the operation of the algorithms is the way that the values $s_{j}$ and $q_{j}$ are determined as a function of the inputs.

\subsection{Complex Exponentiation Algorithm}

In complex exponentiation, the values of $u_{0}$ and $v_{0}$ are set to constants, such as 1 and 0 respectively, while $r_{0}$ and $a_{0}$ are bounded by some intervals. A series of transformations is performed such that $r_{N}$ and $a_{N}$ are constants regardless of the inputs $r_{0}$ and $a_{0}$. From the invariance of 
$T_{j}$, we then have

$$
u_{N}+v_{N} \cdot i=\left(u_{0}+v_{0} \cdot i\right) \times b^{\left(r_{0}+a_{0} \cdot i-r_{N}-a_{N} \cdot i\right)}
$$

Without loss of generality, assume that $r_{N}=0$ and $a_{N}=0$. Each transformation stage must examine one of the values of $r_{j}$ and $a_{j}$ to determine the values of $s_{j}$ and $q_{j}$. Since the goal is to bring $r_{j} \rightarrow r_{N}$ and $a_{j} \rightarrow a_{N}$, this is achieved in a series of stages each of which attempts to satisfy $r_{j+1} \approx r_{N}$ and $a_{j+1} \approx a_{N}$ to an increasing degree of precision in successive stages. Substituting these goals into (8) and (14) leads to the digit selection functions:

$$
\begin{gathered}
s_{j} \approx b^{r_{j}-r_{N}}-1 \\
q_{j} \approx \tan \left(\ln (b) \times\left(a_{j}-a_{N}\right)\right)
\end{gathered}
$$

\subsection{Complex Logarithm Algorithm}

In complex logarithm, the values of $r_{0}$ and $a_{0}$ are constants, the values of $u_{0}$ and $v_{0}$ are inputs, and the series of transformations is performed such that $u_{N}$ and $v_{N}$ are constants. The invariance of $T_{j}$ leads to eqn. (20).

$$
r_{N}+a_{N} \cdot i=\log _{b}\left(\frac{u_{0}+v_{0} \cdot i}{u_{N}+v_{N} \cdot i}\right)+r_{0}+a_{0} \cdot i
$$

A useful choice is $u_{N}=1, v_{N}=0, r_{0}=0$, and $a_{0}=0$. As with the exponentiation algorithm, there is no loss in generality in assuming $u_{N} \neq 0$ and $v_{N}=0$.

To insure that $u_{N}$ and $v_{N}$ are constants, the transformation stages must examine $u_{j}$ and $v_{j}$ to determine $s_{j}$ and $q_{j}$. In this case, the goals are $u_{j} \approx u_{N}$ and $v_{j} \approx v_{N}$. The digit selection functions are potentially more complex, since both scaling and rotation affect both $u_{j}$ and $v_{j}$. To reduce the complexity of digit selection, each function depends only on one of $u_{j}$ and $v_{j}$. For the particular case described above, we require that $s_{j}$ depend on $u_{j}$ alone, and $q_{j}$ depend on $v_{j}$ alone. By setting $u_{j+1} \approx u_{N}$ in (6), and $v_{j+1} \approx v_{N}$ into (12), we have the digit selection functions:

$$
s_{j} \approx \frac{u_{N}}{u_{j}}-1
$$

$$
q_{j} \approx \frac{v_{j}-v_{N}}{u_{j}}
$$

$q_{j}$ depends on two variables, so a constant approximation to $u_{j}$ will be introduced later to simplify the function to a single argument.

\subsection{CORDIC for CLNS Addition}

The CLNS addition function can be constructed using a CORDIC exponentiation, adding one, followed by a CORDIC log, as illustrated in the left side of Fig. 1. Given $\left\langle r_{0}, a_{0}\right\rangle$ as input, a series of exponential stages, with input $\left\langle 1,0, r_{0}, a_{0}\right\rangle$ produces output $\left\langle u_{n}, v_{n}, 1,0\right\rangle$. An adder then produces $u_{0}^{\prime}=u_{n}+1$ and $v_{0}^{\prime}=v_{n}$ and performs a logarithm operation on $\left\langle u_{0}^{\prime}, v_{0}^{\prime}, 0,0\right\rangle$, producing the final result.

An optimization is possible by considering an intermediate value in the exponential stage, say $j$, where $b^{r}$ is represented by $\left(u_{j}+v_{j} \cdot i\right) \times b^{r_{j}+a_{j} \cdot i}$. For sufficiently small $r_{j}$ and $a_{j}$, apply a Taylor series approximation to $\log _{b}\left(1+b^{r}\right)$ producing

$$
\begin{aligned}
& \log _{b}\left(1+b^{r}\right)=\log _{b}\left(1+\left(u_{j}+v_{j} \cdot i\right) \times b^{r_{j}+a_{j} \cdot i}\right) \\
& \log _{b}\left(1+b^{r}\right)=\log _{b}\left(b^{-\left(r_{j}+a_{j} \cdot i\right)}+u_{j}+v_{j} \cdot i\right) \\
& +r_{j}+a_{j} \cdot i \\
& \log _{b}\left(1+b^{r}\right) \approx \log _{b}\left(1-\ln (b) \times\left(r_{j}+a_{j} \cdot i\right)\right. \\
& \left.\quad+u_{j}+v_{j} \cdot i\right)+r_{j}+a_{j} \cdot i
\end{aligned}
$$

For the case that $\ln (b)=1$, the multiply disappears, and circuit uses $r_{0}^{\prime}=1-r_{j}+u_{j}, \quad v_{0}^{\prime}=a_{j}+v_{j}$, $r_{0}^{\prime}=r_{j}$, and $a_{0}^{\prime}=a_{j}$ as inputs to the logarithm stages. This provides a strong incentive to using a base of $e$.

Similar improvements apply to the logarithm stage. Consider some stage $j$, and the Taylor series approximation for $u_{j}^{\prime} \approx 1$ and $v_{j}^{\prime} \approx 0$

$$
\begin{aligned}
& \log _{b}\left(\left(u_{j}^{\prime}+v_{j}^{\prime} \cdot i\right) \times b^{r_{j}^{\prime}+a_{j}^{\prime} \cdot i}\right) \approx \\
& \frac{1}{\ln (b)} \times\left(u_{j}^{\prime}-1+v_{j}^{\prime} \cdot i\right)+r_{j}^{\prime}+a_{j}^{\prime} \cdot i
\end{aligned}
$$


Approximately half the stages can be eliminated for both exponential and logarithm, as shown in the right side of Fig. 1. Consequently, a CLNS addition can be performed with cost comparable to approximately one CORDIC operation.

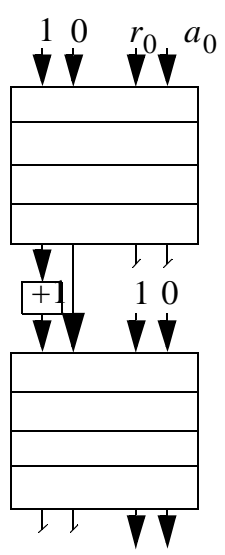

Straightforward Implementation

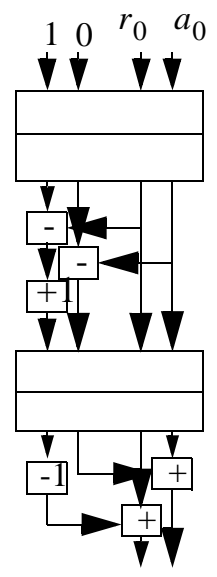

Use of Taylor series
Figure 1 CLNS Addition Using Interpolation. Right half shows truncation of iterations using Taylor series approximation.

\section{Hardware Implementation}

To understand the calculation of the values of $s_{j}$ and $q_{j}$, as well as the bounds on the values and precision in each stage of the computation it is useful at this point to introduce the redundant computation in terms of the hardware implementation.

The algorithms allow redundant representation using of all the quantities involved in the computation; however, the multiplications of $u_{j}$ and $v_{j}$ eliminate any advantage to using a redundant representation of these quantities. Instead, only $r_{j}$ and $a_{j}$ are represented using a carry-save form, and the non-redundant value is calculated to the accuracy required.

Truncation is explicitly represented in the hardware designs shown below with a truncation operator \lfloor\rfloor , and a separate symbol for the truncated value. The precision of any variable in the algorithm, for example some variable $w$, will be expressed as $P(w)$. The precision of a variable is the negative of the position in the binary representation of the least significant bit, i.e., if $w=w_{I-1} \ldots w_{0} \cdot w_{-1} \ldots w_{-F}$, then $P(w)=F$.

There are four related hardware blocks, corresponding to the scaling and rotation stages for both the exponentiation and logarithm algorithms. For both algorithms, the values $u_{j}, v_{j}, r_{j}$, and $a_{j}$ are assumed to have $F$ bits precision. Internally, each value is represented with an additional $G$ guard bits, for a total of $F+G$ bits. In carry-save form, $r_{j}$ and $a_{j}$ are represented by the pairs $r c_{j}$ and $r s_{j}$, and by $a c_{j}$ and $a s_{j}$ respectively, all of which have precision $F+G$.

In the exponential scaling stage, shown in Figure 2, a reduced precision approximation of $r_{j}$ is calculated by truncating $r c t_{j}$ and $r s t_{j}$, which are added to calculate the non-redundant, but lower accuracy approximation $r t_{j}$, which is input to the digit calculation block. The purpose of this lower precision approximation is to reduce the amount of hardware required for the adder, but more importantly, to reduce the number of bits that the digit selection block must examine, and consequently reduce its associated hardware and increase its speed.

All stages described here contain digit selection blocks. Before describing the specific functions implemented in them, a brief description of their logic structure is useful. A digit selection block implements some monotonic function of a single input, and is a piecewise constant approximation to some continuous function. Using the specific example of a digit selection block with $r t_{j}$ as an input and $s_{j}$ as output, with $s_{j}=f\left(r t_{j}\right)$ for some $f()$, the function can be expressed in the form (27), where the digit is $k$ and $k \times 2^{-P\left(s_{j}\right)}$ is the piecewise constant approximation to the function over some interval $\left[\right.$ thresh $_{k}$, thresh $_{k+1}$ ).

$$
\begin{gathered}
s_{j}=k \times 2^{-P\left(s_{j}\right)} \text { thresh }_{k} \leq r t_{j}<\text { thresh }_{k+1}, \\
k_{\text {min }} \leq k \leq k_{\text {max }} \\
\text { thresh }_{k}=f^{-1}\left(\left(k-\frac{1}{2}\right) \times 2^{-P\left(s_{j}\right)}\right)
\end{gathered}
$$

The value of $k$ may take any value in the range $k_{\text {min }}$ to $k_{\text {max }}$, where the bounds are chosen to include the entire range of inputs to the function. The number of distinct values that can be produced is $k_{\text {max }}-k_{\text {min }}+1$ and is referred to as the radix of the value $r t_{j}$. Expressing the function at this level of detail explicitly provides the range over which each input value produces some output value, and makes it straightforward to bound the result of a calculation. The 
hardware implementation of this will be discussed later, but it is clear that the two primary factors involved are the number of bits in the input value which need to be examined, and the number of distinct output values that can be produced, or equivalently, the radix.

The digit selection block in the scaling exponentiation stage produces $s_{j}$ and $l_{j}=-\log _{b}\left(1+s_{j}\right)$. Assuming that the goal is $r_{N}=0$, we desire that $l_{j} \approx-r_{j}$ to the precision possible given the fixed number of bits in the representation of $s_{j}$. Note that $l_{j}$ is expressed to the full precision of the datapath, while it is the limited number of bits in $s_{j}$ that restrict the set of possible values of $l_{j}$. This is due to the desire to reduce the size of the digit estimation logic for $s_{j} \cdot$

The values of $u_{j}$ and $v_{j}$ are also truncated to a lower precision forming $u t_{j}$ and $v t_{j}$, and a pair of multiplyadders is used to calculate the results specified in Eqns. (6) and (7). The multiplier output is left in carry-save form, which avoids a carry-propagate addition inside it. This is advantageous as the result of the multiplier is later added using a CSA and CPA. The multiplier result is also truncated to the precision of $u_{j}$ and $v_{j}$. Eqn (8) is calculated using a CSA because of the redundant representation of $r_{j}$.

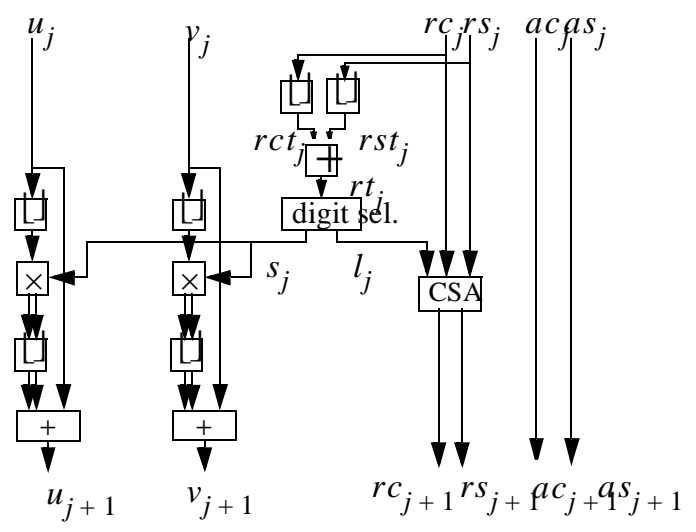

Figure 2 Scaling Stage for Exponentiation

Figure 3 illustrates the hardware for the rotation stage for exponentiation. A reduced precision approximation to $a_{j}$ is calculated and input to the digit calculation block. This produces $q_{j}$ and is used, together with reduced precision values of $u_{j}$ and $v_{j}$ to perform the calculation in
Eqns. (11) through (14). As before, calculations involving $r_{j}$ and $a_{j}$ are preformed with CSAs.

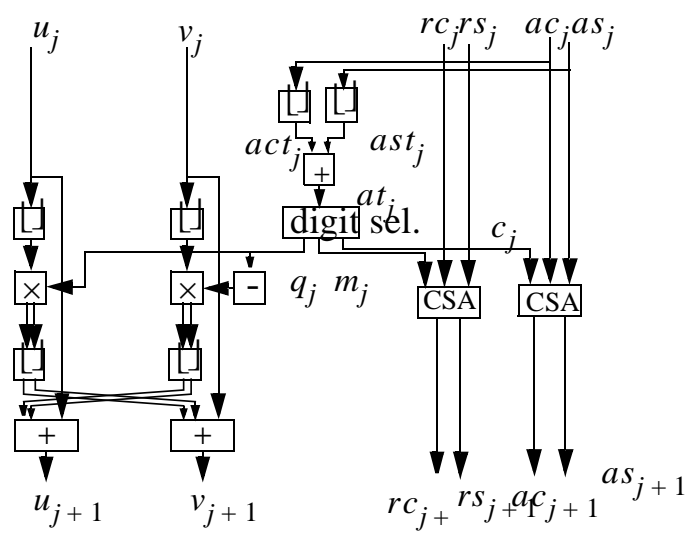

Figure 3 Rotation Stage for Exponentiation

Figures 4 and 5 illustrate the rotation and scaling stages for the logarithm algorithm. These are similar to the previous two, except that $u_{j}$ and $v_{j}$ are used for determining $s_{j}$ and $q_{j}$ respectively. This complicates the analysis of the bounds on the intermediate results, as each digit selection block can only access part of the problem state.

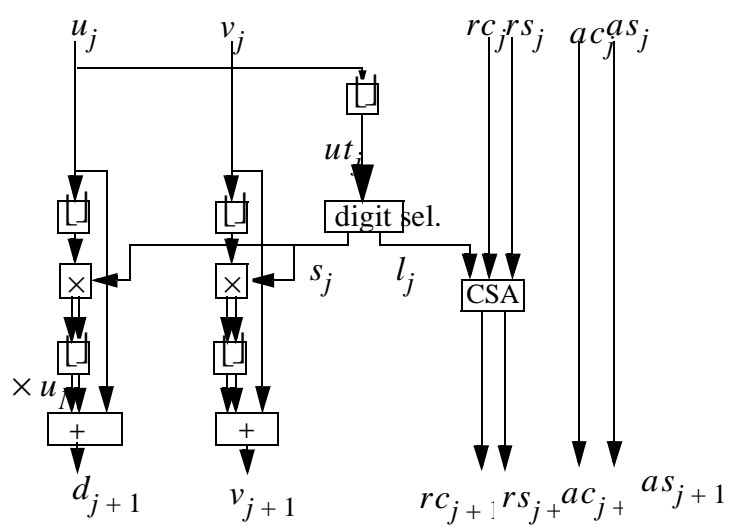

Figure 4 Scaling Stage for Logarithm

\section{Bounds on Intermediate Results}

Antelo et al advocate high-radix CORDIC using a maximally redundant digit set. They suggest choosing a radix, and show that the algorithm will converge using the maximally redundant digit set. In this paper, we suggest explicit calculation of the minimal possible redundant digit set, and exact digit selection based on the truncated data for two reasons First, explicitly calculating the radix means that the 


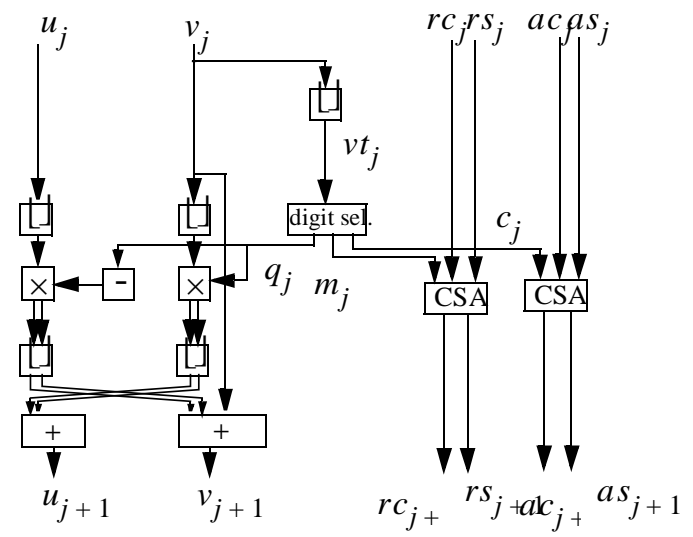

Figure 5 Rotation Stage for Logarithm

tables need only be large enough to accommodate the actual number of values, which can be smaller than the radix implied by the maximally redundant digit set. Second, the size of the multipliers can potentially be reduced if the set of values to be multiplied is bounded by a smaller range than the representation allows. The latter of these depends on the observation that the core of an $n$-bit modified Booth multiplier value can be used to multiply values in the range $\left[-\frac{5}{4} \times 2^{n-1}, \frac{5}{4} \times 2^{n-1}-1\right]$. This range would conventionally require an $n+2$ bit multiplier. For example, multiplication by integers in $[-40,39]$ can be performed with a 6-bit multiplier, but would conventionally require an 8-bit multiplier.

The approach in this section is constructive. We define the bounds of the operands at each stage, and determine the relationship between the bound on the input and output for each type of transformation. For each possible digit $k$, the range of inputs that use the digit are specified, and the resulting bound on the output is determined. Given the overall bound on the range of inputs, and the precision of the digits $s_{j}$ and $q_{j}$, it is possible to determine the set of values $k$ required to span the entire input range. and consequently determine the radix of the digit set. By computing the union of all output bounds for every possible digit in a stage, an overall bound on the output of a stage can be calculated. It is also necessary to specify the precision of the truncated quantities input to the digit selection logic.

It will be useful to have a concise notation for the truncation or rounding of quantities to various fixed point precisions. We use $\lfloor x\rfloor_{P}$ to mean the value of $x$ truncated down to $P$ bits precision, so the definition is given as:

$$
\lfloor x\rfloor_{P}=\left\lfloor x \times 2^{P}\right\rfloor \times 2^{-P}
$$

Similar notation for rounding up, and round to nearest are also used:

$$
\begin{gathered}
\lceil x\rceil_{P}=\left\lceil x \times 2^{P}\right\rceil \times 2^{-P} \\
{[x]_{P}=\left\lfloor x \times 2^{P}+2^{P-1}\right\rfloor \times 2^{-P}}
\end{gathered}
$$

It is also necessary to bound the variables at each stage. We introduce $\min$ and $\max$ subscripts such that any variable is bounded by its $\min$ and $\max$ as in $u_{\min , j} \leq u_{j}<u_{\max , j}$.

\subsection{Scaling Stage for Exponential}

As mentioned, it is desirable that $l_{j} \approx r_{j}$, or equivalently, $s_{j} \approx b^{r}-1$. Ideally, the digit selection function would implement

$$
s_{j}=\left[b^{r{ }_{j}}-1\right] P\left(s_{j}\right)
$$

Transforming this into the piecewise-constant expression leads to the exact computation of the digit $s_{j}$ :

$$
\begin{aligned}
& s_{j}=k \times 2^{-P\left(s_{j}\right)} \\
& \log _{b}\left(1+\left(k-\frac{1}{2}\right) \times 2^{-P\left(s_{j}\right)}\right) \leq r t_{j} \\
& <\log _{b}\left(1+\left(k+\frac{1}{2}\right) \times 2^{-P\left(s_{j}\right)}\right) \\
& k_{\text {min }} \leq k \leq k_{\text {max }}
\end{aligned}
$$

The range of values of $k$ is required to be sufficiently large that all possible values of $r t_{j}$ will fall in one such interval. The truncation of the carry save value of $r_{j}$ bounds on $r t_{j}$ :

$$
r_{j}-2^{1-P\left(r t_{j}\right)}+2^{1-F-G} \leq r t_{j} \leq r_{j}
$$

Substituting (33) into produces constraints for $k_{\text {min }}$ and $k_{\text {max }}$, which can be further simplified into explicit requirements for these two bounds on $k$ :

$$
\begin{aligned}
& \log \left(1+\left(k_{\min }-\frac{1}{2}\right) \times 2^{-P\left(s_{j}\right)}\right) \\
& +2^{1-P\left(r_{j}\right)}-2^{1-F-G} \leq r_{\text {min }, j}
\end{aligned}
$$




$$
\log \left(1+\left(k_{\text {max }}+\frac{1}{2}\right) \times 2^{-P\left(s_{j}\right)}\right) \geq r_{\text {max }, j}
$$

Note that in these equations the rounding of $\log _{b}()$ to $F+G$ precision is not specified; however, whatever method is used must be applied consistently across all of these equations to obtain correct results.

Bounds on $r_{j+1}$ are calculated by first noting that $r t_{j}$ is bounded by (33) due to the truncation of the carry and sum components from $F+G$ precision before their addition. For each such value of $k$ that is required, it is possible to use (33) to determine the bounds on $r_{j}$

$$
r t_{j} \leq r_{j} \leq r t_{j}+2^{1-P\left(r t_{j}\right)}-2^{1-F-G}
$$

The definition of $s_{j}$ is then substituted into (8) and (10) to produce bounds for each given value of $k$ :

$$
\begin{gathered}
\log _{b}\left(1+\left(k-\frac{1}{2}\right) \times 2^{-P\left(s_{j}\right)}\right) \\
-\log _{b}\left(1+k \times 2^{-P\left(s_{j}\right)}\right) \leq r_{j+1} \\
r_{j+1}<\log _{b}\left(1+\left(k+\frac{1}{2}\right) \times 2^{-P\left(s_{j}\right)}\right)+2^{1-P\left(r t_{j}\right)} \\
-2^{1-F-G}-\log _{b} 1+k \times 2^{-P\left(s_{j}\right)}
\end{gathered}
$$

It is possible to attempt to construct an explicit bound for all possible $k$ by substituting in the appropriate $k_{\min }$ and $k_{\text {max }}$, but it is difficult to guarantee an exact bound in the presence of multiple roundings to various precisions. Instead, we simply iterate across all values, taking the minimum and maximum of these bounds to determine overall bounds on $r_{j+1}$

It is useful to ignore the redundancy and take a first order Taylor series approximation to (37) and (38) into obtain insight into the operation of the algorithm, although the exact form of equation must be used for computing bounds.

$$
\left|r_{j+1}\right| \leq \ln (b) \times 2^{-1-P\left(s_{j}\right)}
$$

Eqn (39) shows that each stage reduces the magnitude of $r_{j+1}$ to roughly one-half ULP in the digit's representation.

\subsection{Rotation Stage for Exponentiation}

A similar approach can be taken for the rotation stage, with the notable difference that the rotation is computed as a function of $a_{j}$, but affects both $a_{j+1}$ and $r_{j+1}$. In the rotation stage, we desire $q_{j}=\left[\tan \left(\ln (b) \times a_{j}\right)\right]_{P\left(q_{j}\right)}$.

The piecewise constant approximation is given as

$$
\begin{aligned}
& q_{j}=k \times 2^{-P\left(q_{j}\right)} \\
& \frac{\operatorname{atan}\left(\left(k-\frac{1}{2}\right) \times 2^{-P\left(q_{j}\right)}\right)}{\ln (b)} \leq a t_{j} \\
& <\frac{\operatorname{atan}\left(\left(k+\frac{1}{2}\right) \times 2^{-P\left(q_{j}\right)}\right)}{\ln (b)} \\
& k_{\text {min }} \leq k \leq k_{\text {max }}
\end{aligned}
$$

Exact bounds on $k$ can be determined by the previous approach and will not be presented.

Bounds on $a_{j+1}$ can be found by a similar approach to the scaling stage. This leads to the following bounds on $a_{j+1}$, where the union of all such bounds must be taken for the entire range of $k$

$$
\begin{gathered}
\frac{\operatorname{atan}\left(\left(k-\frac{1}{2}\right) \times 2^{-P\left(q_{j}\right)}\right)}{\ln (b)} \\
-\frac{\operatorname{atan}\left(k \times 2^{-P\left(q_{j}\right)}\right)}{\ln (b)} \leq a_{j+1} \\
a_{j+1}<\frac{\operatorname{atan}\left(\left(k+\frac{1}{2}\right) \times 2^{-P\left(q_{j}\right)}\right)}{\ln (b)} \\
-\frac{\operatorname{atan}\left(k \times 2^{-P\left(q_{j}\right)}\right)}{\ln (b)}+2^{1-P\left(a t_{j}\right)}-2^{1-F-G}
\end{gathered}
$$

For small $a_{j}$, ignoring redundancy and taking a firstorder Taylor series approximation shows that $a_{j+1}$ is bounded by approximately half an ULP of $q_{j}$.

$$
\left|a_{j+1}\right|<\frac{2^{-1-P\left(q_{j}\right)}}{\ln (b)}
$$

The choice of $q_{j}$ is independent of $r_{j}$, so the bound on $r_{j+1}$ can only be given by subtracting the minimum and 
maximum possible values of $m_{j}$ from the bounds on $r_{j}$,

$$
\begin{aligned}
& r_{\min , j}-\max _{k}\left(\log _{b}\left(\sqrt{1+k^{2} \times 2^{-2 \times P\left(a t_{j}\right)}}\right)\right) \leq r_{j+1} \\
& r_{j+1} \leq r_{\max , j}-\min _{k}\left(\log _{b}\left(\sqrt{1+k^{2} \times 2^{-2 \times P\left(a t_{j}\right)}}\right)\right)
\end{aligned}
$$

Because $k$ may be signed, it is not possible to express these in a form only using one of $k_{\text {min }}$ or $k_{\max }$ in each equation.

\subsection{Scaling Stage for Logarithm}

The logarithm stages are more complicated to understand as both scaling and rotation affect both $u_{j+1}$ and $v_{j+1}$, but each stage must perform a transformation based on only one value. Fig. 6 illustrates the scaling transformation. The vertical dashed lines represent the bounds between values of $u_{j}$ that lead to distinct values of $s_{j}$. Each such bound leads to an interval in $u_{j}$ and $v_{j}$ that is a rectangular interval subject to scaling by the same value of $1+s_{j}$. The arrows show the linear scaling by $1+s_{j}$ for the upper left point in two of the rectangles. Although the range of $u_{j+1}$ is reduced compared to the range of $u_{j}$, the range of $v_{j+1}$ is increased compared to $v_{j}$.

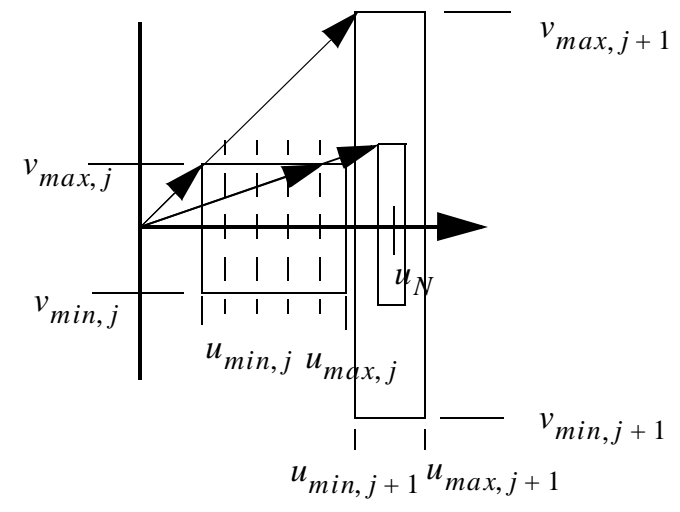

Figure 6 Operation of Scaling Stage in Logarithm Algorithm

Recall that we desire $s_{j} \approx \frac{u_{N}}{u_{j}}-1$. In the piecewise constant form, this can be achieved by

$$
s_{j}=k \times 2^{-P\left(s_{j}\right)}
$$

$$
\begin{gathered}
\frac{u_{N}}{\left(k+\frac{1}{2}\right) \times 2^{-P\left(s_{j}\right)}+1} \leq u t_{j}<\frac{u_{N}}{\left(k-\frac{1}{2}\right) \times 2^{-P\left(s_{j}\right)}+1}, \\
k_{\text {min }} \leq k \leq k_{\max }
\end{gathered}
$$

This establishes following bound on $u_{j+1}$. Although $k=k_{\min }$ will give the largest range, the calculation should be performed to determine the union of all ranges for all values of $k$ using exactly the same rounding as the hardware in order to obtain precise bounds.

$$
\begin{gathered}
u_{N} \times \frac{k \times 2^{-P\left(s_{j}\right)}+1}{\left(k+\frac{1}{2}\right) \times 2^{-P\left(s_{j}\right)}+1} \leq u_{j+1} \\
u_{j+1}<\left(\frac{u_{N}}{\left(k-\frac{1}{2}\right) \times 2^{-P\left(s_{j}\right)}+1}\right. \\
\left.+2^{1-P\left(u t_{j}\right)}-2^{1-F-G}\right) \times\left(k \times 2^{-P\left(s_{j}\right)}+1\right)
\end{gathered}
$$

The value of $v_{j}$ is scaled by the same factor, so $v_{j+1}$ can be bounded by the union of the intervals for all values of $k$

$$
\begin{aligned}
& v_{\text {min }, j} \times\left(k \times 2^{-P\left(s_{j}\right)}+1\right) \leq v_{j+1} \\
& <v_{\text {max }, j} \times\left(k \times 2^{-P\left(s_{j}\right)}+1\right)
\end{aligned}
$$

\subsection{Rotation Stage for Logarithm}

The rotation stage for logarithm presents the most difficulty, as the goal $q_{j} \approx \frac{v_{j}}{u_{j}}$ depends on two values. We simplify this to a function of a single value by using the bound on $u_{j}$ and taking the midpoint of this range as an approxi-

mation. This results in $q_{j}=\left[\frac{v_{j}}{\left(\frac{u_{\min , j}+u_{\max , j}}{2}\right)}\right]_{P\left(q_{j}\right)}$ as an approximate goal for $q_{j}$. This operation of this stage is shown graphically in Fig. 7. The horizontal dashed lines represent the thresholds for values of $v_{j}$ that result in dis- 
tinct values of $q_{j}$, and that apply to a rectangular region in $u_{j}$ and $v_{j}$. The figure shows the rotation and stretching of two of these regions, illustrating the center point of each, together with the bounding box for the rotated and stretched rectangle. Because each of the intervals is rotated, the tightest possible bounds of the resulting values $u_{j+1}$ and $v_{j+1}$ does not form a rectangle; however, for simplicity of analysis, it is considered to be the smallest rectangle that encloses all of the rotated and stretched intervals, and is illustrated with the dotted rectangle in the Fig. 7. The figure is not to scale, and typically the rectangle bounding the would be much smaller than the input bounds.

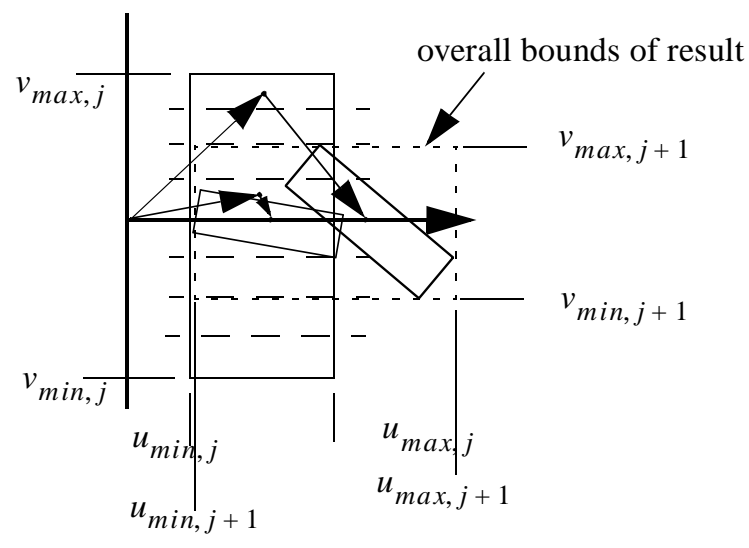

Iigure 7 Operation of Rotation Stage in Logarithm Algorithm

The exact definition of $q_{j}$ is

$$
\begin{gathered}
q_{j}=k \times 2^{-P\left(q_{j}\right)} \\
\left(k-\frac{1}{2}\right) \times 2^{-P\left(q_{j}\right)} \times\left(\frac{u_{\text {min }, j}+u_{\text {max }, j}}{2}\right) \leq v t_{j} \\
<\left(k+\frac{1}{2}\right) \times 2^{-P\left(q_{j}\right)} \times\left(\frac{u_{\text {min }, j}+u_{\text {max }, j}}{2}\right) \\
k_{\text {min }} \leq k \leq k_{\text {max }}
\end{gathered}
$$

This leads to the following bounds on $u_{j+1}$ and $v_{j+1}$, where, as usual, all values of $k$ must be considered:

$$
\begin{aligned}
& \left(k-\frac{1}{2}\right) \times 2^{-P\left(q_{j}\right)} \times\left(\frac{u_{\min , j}+u_{\max , j}}{2}\right) \\
& -k \times 2^{-P\left(q_{j}\right)} \times u_{\max , j} \leq v_{j+1}
\end{aligned}
$$

$$
\begin{gathered}
v_{j+1}<\left(k+\frac{1}{2}\right) \times 2^{-P\left(q_{j}\right)} \times\left(\frac{u_{\text {min }, j}+u_{\text {max }, j}}{2}\right) \\
+2^{1-P\left(v t_{j}\right)}+2^{1-F-G}-k \times 2^{-P\left(q_{j}\right)} \times u_{\text {min }, j} \\
u_{\text {min }, j}+k \times 2^{-P\left(q_{j}\right)} \times v_{\text {min }, j} \leq u_{j+1} \\
u_{j+1}<u_{\text {max }, j}+k \times 2^{-P\left(q_{j}\right)} \times v_{\text {max }, j}
\end{gathered}
$$

\section{Example for 32-bit Complex Numbers}

The exponential stages calculate both digits based on a single operand, but the value of $a_{j}$ affects the value of $r_{j+1}$ as well. It is optional whether to interleave rotation and scaling stages, or to perform all of the scaling at the end, as in [9]. In the logarithm stage, both $u_{j+1}$ and $v_{j+1}$ depend on $u_{j}$ and $v_{j}$. It is necessary to alternate scaling and rotation stages in order to tighten bounds on both simultaneously.

As a demonstration of the feasibility of this approach, we have designed a high-radix multiply-add CLNS arithmetic unit with precision comparable to IEEE-754 single precision. The unit performs complex multiplication using two fixed point adders, and uses high-radix exponentiation and logarithm to perform complex addition using the function $f(r)$ defined in (4). The design uses $F=24$ and $G=4$, and some other minor changes to the constants assumed in the derivation above. The number representation uses a mixed base for the representation of the numbers to simplify range reduction. $\mathrm{A}$ number $\mathrm{X}$ is represented by its complex logarithm $x=x_{L}+x_{\theta} \cdot i$, where $x_{L}$ is a 32-bit 2's complement fixed point number, and $x_{\theta}$ is a 27-bit unsigned fixed point number, both of which have 24 fractional bits.

The details of the algorithm were designed with the assistance of a program that has as input an architecture description file containing all of the precisions of each variable, and performs exact bit-level modeling of the architecture.

Our design uses a total of 10 stages to perform an exponentiation and a logarithm as required by the logarithmic addition function. Datapath widths were based on 6 bit digits requiring two stages of each of rotation and scaling for exponentiation, and three of each for logarithm. Antelo et al [9] would require 8 stages using 7-bit multipliers to perform a rotation to the same precision; thus, our architecture requires little more hardware to perform a CLNS addition. 
Beyond this, only two more fixed point adders are required to perform a CLNS multiply-add. Table 1 shows the key parameters of the design. The digit precision refers to the

Table 1: Hardware Parameters of Example CLNS ALU

\begin{tabular}{|c|c|c|c|}
\hline $\begin{array}{c}\text { Stage } \\
\text { Type }\end{array}$ & $\begin{array}{c}\text { Digit } \\
\text { Precision }\end{array}$ & $\begin{array}{c}\text { Digit } \\
\text { Selection } \\
\text { Precision }\end{array}$ & Radix \\
\hline \hline \multicolumn{4}{|c|}{ Exponential Algorithm } \\
\hline R & 6 & 10 & 65 \\
\hline S & 6 & 10 & 118 \\
\hline R & 12 & 16 & 74 \\
\hline S & 12 & 16 & 77 \\
\hline \multicolumn{5}{|c|}{ Logarithm Algorithm } \\
\hline S & 6 & 10 & 65 \\
\hline R & 6 & 10 & 65 \\
\hline S & 6 & 10 & 67 \\
\hline R & 12 & 16 & 82 \\
\hline S & 12 & 16 & 69 \\
\hline R & 18 & 22 & 110 \\
\hline
\end{tabular}

digit generated, either $s_{j}$ or $p_{j}$, and the digit selection precision refers to the input to the digit selection block, such as $a t_{j}$ or other values.

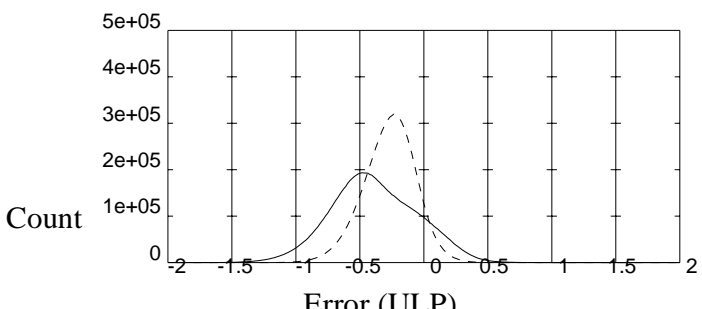

Figure 8 Frequency Count of Error of CLNS ALU Example: Solid line: real; Dashed: Imaginary. Bin width is .01

Fig. 8 illustrates the simulator's real and imaginary error histogram in ULPs for $10^{7}$ pseudo-random tests. A bias is clear, and is due to the use of truncation rather than rounding in the datapath. Mean error and bias are each less than 0.4 ULP, but worst case error is 1.5 ULP.

\section{Conclusions}

This paper has demonstrated high-radix CORDIC algorithms adapted for CLNS addition. A design example producing six bits per stage as an illustration shows that a CLNS addition can be performed for approximately the same cost as a conventional high-radix CORDIC rotation. Since a CLNS multiply is inexpensive, this allows a CLNS multiply-accumulate to be performed for the cost of a sin- gle CORDIC operation.

\section{References}

[1] J. Volder, "The CORDIC Computing Technique", IRE Trans. Comput., Sept. 1959, pp. 330-334

[2] J. Walther, "A Unified Algorithm for Elementary Functions", Spring Joint Comp. Conf., 1971, pp 379-385

[3] T. Chen, "Automatic Computation of Exponentials, Logarithms, Ratios, and Square Roots", IBM J. Res. Dev, 1972, pp 380-388

[4] A. Madisetti, A. Kwentus, and A. Willson, "A Sine/Cosine Direct Digital Frequency Synthesizer Using an Angle Rotation Algorithm", ISSCC-95, pp. 262-263

[5] A. Skaf, J.-M. Mullar, and A. Guyot, "On-line Hardware Implementation for Complex Exponential and Logarithm", Twentieth European Solid-State Circ. Conf., 1994, pp 252255

[6] D. Timmermannm B. Rix, H. Hahn, and B. Hosticka, "A CMOS Floating-Point Vector Arithmetic Unit", IEEE J. Solid State Circuits, May 1994, pp 634-639

[7] D. Lewis, "A 114 MFLOPS Logarithmic Number System Arithmetic Unit for DSP Applications", IEEE J. Solid-State Circuits, Dec 1995, pp 1547-1553

[8] J.-C. Bajard, S. Kla, and J.-M. Muller, "BKM: A New Hardware Algorithm for Complex Elementary Functions", IEEE Trans. Comput, Aug 1994, pp 955-964

[9] E. Antelo, J. Villalba, J. Bruguera, and E. Zapata, "High Performance Rotation Architectures Based on the Radix-4 CORDIC Algorithm", IEEE Trans. Comput., Aug 1997, pp 855-870.

[10] E. Swartzlander and A. Alexopolous, "The Sign/Logarithm Number System", IEEE Trans. Comput., Dec 1975, pp. 1238-1242

[11] M. Arnold, T. Bailey, J. Cowles, and M. Winkel, "Arithmetic Co-Transformation in the Real and Complex Logarithmic Number Systems", IEEE. Trans. Comput., July 1998, pp 777-786

[12] P. Baker, "Parallel Multiplicative Algorithms for Some Elementary Functions", IEEE Trans. Comput., March 1975, pp 322-325

[13] H. Ahmed, Signal Processing Algorithms and Architectures, PhD Thesis, Stanford University, 1982

[14] H. Ahmed, Efficient Elementary Function Generation with Multipliers, Proc. 9th Symp. Comp. Arith, 1989, pp 52-59

[15] E. Antelo, J. Brugerea, T. Lang, J. Villalba, and E. Zapata, "High Radix Cordic Rotation based on Selection by Rounding", Intl. European Conf. on Parallel Proc., Euro-Par 96, pp 155-164 